\title{
DYNAMICAL SYSTEMS FROM FUNCTION ALGEBRAS
}

\author{
TIM PENNINGS AND JUSTIN PETERS
}

(Communicated by John B. Conway)

\begin{abstract}
Let $X$ be compact Hausdorff, $\Sigma$ the natural numbers or integers, $\varphi: X \rightarrow X$, and $\left\{\varphi^{k}: k \in \Sigma\right\}$ a (semi)group of continuous functions from $X$ to $X$. Given the dynamical system $(X, \varphi, \Sigma)$, let $\mathfrak{A}$ be a $\Sigma$-invariant $C^{*}$ algebra of bounded functions containing $C(X)$. There is a natural extension $(\widehat{X}, \widehat{\varphi}, \Sigma)$ of $(X, \varphi, \Sigma)$ where $\widehat{X}$ is the spectrum of $\mathfrak{A}$ and $\widehat{\varphi}$ is given by $\hat{\varphi}(\hat{x}) f=\hat{x}(f \circ \varphi)$. If $\mathfrak{A}$ has a dense subset of functions continuous on a cofinite set, then $(\widehat{X}, \widehat{\varphi}, \Sigma)$ inherits the properties of minimality and topological transitivity from $(X, \varphi, \Sigma)$ if $\mathfrak{A}$ contains no point characteristic functions.
\end{abstract}

\section{INTRODUCTION}

Let $X$ be a compact Hausdorff space, and take $\Sigma$ to be either the semigroup $N(=\{0,1,2, \ldots\})$ or $\mathbf{Z}$. Let $\varphi: X \rightarrow X$ be a continuous mapping $(\Sigma=$ $N)$ or a homeomorphism $(\Sigma=\mathbf{Z})$, so that $\varphi^{k}$ is continuous for all $k \in \Sigma$. The triple $(X, \varphi, \Sigma)$ is called a dynamical system. To avoid trivialities, we will assume that all compact Hausdorff spaces under consideration have infinite cardinality.

Given the dynamical systems $(X, \varphi, \Sigma)$ and $(Y, \psi, \Sigma)$, if there exists a continuous surjection, $p: Y \rightarrow X$ for which the following diagram commutes,

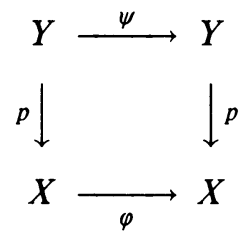

then $(Y, \psi, \Sigma)$ is called an extension of $(X, \varphi, \Sigma)$, while $(X, \varphi, \Sigma)$ is called a factor of $(Y, \psi, \Sigma)$.

Given $x \in X$, the set $\left\{\varphi^{k}(x): k \in \Sigma\right\}$ is called the $\varphi$-orbit of $x$ and is denoted by $\mathscr{O}_{\varphi}(x)$. If there is an $x \in X$ such that $\mathscr{O}_{\varphi}(x)$ is dense in $X$, then

Received by the editors December 18, 1987. Presented at the 94th Annual Meeting of the American Mathematical Society in Atlanta, January 6-9, 1988.

1980 Mathematics Subject Classification (1985 Revision). Primary 43A45; Secondary 34C35, 46L55, 47D05, 70G99.

Key words and phrases. Dynamical system, extension, Gelfand transform, minimal, orbit, spectrum, topologically transitive. 
$(X, \varphi, \Sigma)$ is said to be topologically transitive. If $\mathscr{O}_{\varphi}(x)$ is dense in $X$ for all $x \in X$, then $(X, \varphi, \Sigma)$ is called minimal.

It is easy to show that if $(X, \varphi, \Sigma)$ has either of these dynamical properties, then any factor of $(X, \varphi, \Sigma)$ enjoys the same property. However, these properties will in general fail to carry over to extensions. Most of the discussion in the literature on extensions of dynamical systems concerns extensions arising from a construction like the skew-product [5]; however, it is also natural to study extensions obtained from function algebras.

Let $B(X)$ denote the algebra of all bounded, complex-valued functions on $X$ in the supremum norm, and let $\mathfrak{A}$ be a $C^{*}$-algebra of functions on $X$ with $C(X) \subset \mathfrak{A} \subset B(X)$ and such that $f \circ \varphi^{k} \in \mathfrak{A}$ whenever $f \in \mathfrak{A}, k \in \Sigma$. (We say $\mathfrak{A}$ is $\Sigma$-invariant.) If $\widehat{X}$ is the spectrum of $\mathfrak{A}$, then $\widehat{X}$ is compact and with the map $\hat{\varphi}: \widehat{X} \rightarrow \hat{X}$ defined by $\hat{\varphi}(\hat{x}) f=\hat{x}(f \circ \varphi),(\hat{X}, \widehat{\varphi}, \Sigma)$ is a dynamical system. Furthermore, this dynamical system is an extension of $(X, \varphi, \Sigma)$; the map $p: \widehat{X} \rightarrow X$ is simply taken to be the restriction of pure states of $\mathfrak{A}$ to $C(X)$. Generally speaking, the larger the algebra $\mathfrak{A}$, the less the resemblance between $X$ and $\hat{X}$.

For example, let $\mathscr{B}$ denote the $C^{*}$-algebra generated by the bounded, complex-valued functions on $X$ which are continuous on a cofinite set (i.e., the complement of a set of finite cardinality). (Of course $\mathscr{B}$ will also contain functions with infinitely many points of discontinuity). Suppose that $(X, \varphi, \Sigma)$ is minimal and $\varphi$ has the property that $\varphi^{-1}(x)$ is of finite cardinality for each $x \in X$. (Then $f \circ \varphi^{k} \in \mathscr{B}$ whenever $f \in \mathscr{B}$.) Taking $\mathfrak{A}=\mathscr{B}$, $(\widehat{X}, \widehat{\varphi}, \Sigma)$ will not even be topologically transitive. For given $x_{1} \in X$, there is an $x_{0} \in X \backslash \mathscr{O}_{\varphi}\left(x_{1}\right)$. We will see that the function

$$
e_{x_{0}}= \begin{cases}1, & x=x_{0} \\ 0, & x \neq x_{0}\end{cases}
$$

gives rise to an isolated point in $\widehat{X} \backslash \mathscr{O}_{\hat{\varphi}}\left(\hat{x}_{1}\right)$ where $\hat{x}_{1} \in p^{-1}\left(x_{1}\right)$.

Our principal result (Theorem II.9) includes that if $\mathfrak{A}$ has a dense subset of functions each of which is continuous on a cofinite set and if $\mathfrak{A}$ contains no function $e_{x}$, then $(\widehat{X}, \widehat{\varphi}, \Sigma)$ inherits the properties of minimality and topological transitivity from $(X, \varphi, \Sigma)$.

II

We continue with the notation from $I$.

II.1. Lemma. The map $\widehat{\varphi}: \widehat{X} \rightarrow \widehat{X}$ satisfies

(i) $\hat{\varphi}$ is continuous;

(ii) if $\varphi$ is surjective, then $\hat{\varphi}$ is surjective,

(iii) if $\varphi$ is a homeomorphism, then $\widehat{\varphi}$ is a homeomorphism; 
(iv) $p: \widehat{X} \rightarrow X$ is a continuous surjection and the diagram

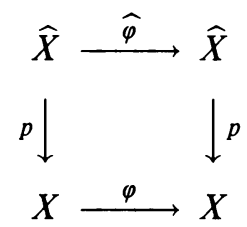

commutes. Thus, the dynamical system $(\widehat{X}, \widehat{\varphi}, \Sigma)$ is an extension of $(X, \varphi, \Sigma)$.

The proofs are routine.

Next we give an alternative description of $\widehat{X}=\operatorname{spec}(\mathfrak{A})$. Let

$$
Y=\operatorname{cl}\left\{\left(x,(f(x))_{f \in \mathfrak{A}}\right): x \in X\right\} \subset X \times \prod_{f \in \mathfrak{A}} \overline{f(X)} ;
$$

so $Y$ is compact Hausdorff.

Define the maps $p: Y \rightarrow X$ and $p_{f}: Y \rightarrow \overline{f(X)} \subset \mathbf{C}$ as follows: if $y=\left(x,\left(t_{f}\right)_{f \in \mathfrak{A}}\right)$, let $p(y)=x, p_{f}(y)=t_{f}$. Then $p, p_{f}$ are continuous surjections.

Now the maps $p_{f}$ separate the points of $Y$ : For suppose $y_{1} \neq y_{2}$ where $y_{1}=\left(x_{1},\left(t_{f}\right)_{f \in \mathfrak{A}}\right), y_{2}=\left(x_{2},\left(s_{f}\right)_{f \in \mathfrak{A}}\right)$. If $x_{1} \neq x_{2}$, then there is a continuous function $f_{0} \in C(X) \subset \mathfrak{A}$ for which $f_{0}\left(x_{1}\right) \neq f_{0}\left(x_{2}\right)$. Since $t_{f_{0}}=f_{0}\left(x_{1}\right)$ and $s_{f_{0}}=f_{0}\left(x_{2}\right)$, it follows $p_{f_{0}}\left(y_{1}\right) \neq p_{f_{0}}\left(y_{2}\right)$. If $x_{1}=x_{2}$, then for some $f_{1} \in \mathfrak{A}$, $t_{f_{1}} \neq s_{f_{1}}$, so $p_{f_{1}}\left(y_{1}\right) \neq p_{f_{1}}\left(y_{2}\right)$.

Thus the set $\left\{p_{f}: f \in \mathfrak{A}\right\}$ is an algebra of continuous, complex-valued functions on $Y$ which separates the points, contains the constant functions, and is closed under complex conjugation. Furthermore,

$$
\begin{aligned}
\left\|p_{f}\right\| & =\sup \left\{\left|p_{f}(y)\right|: y \in Y\right\}=\sup \{|t|: t \in \overline{f(X)}\} \\
& =\sup \{|f(x)|: x \in X\}=\|f\| .
\end{aligned}
$$

It follows that $f \rightarrow p_{f}$ is an isometric isomorphism of $\mathfrak{A}$ with a dense subalgebra of $C(Y)$ (by Stone-Weierstrass). But $\mathfrak{A}$ is complete by hypothesis, so $\left\{p_{f}: f \in \mathfrak{A}\right\}$ is complete and hence equal to $C(Y)$. This proves the

\section{II.2. Proposition. $Y=\operatorname{spec}(\mathfrak{A})$.}

Henceforth we will often use $\hat{f}$ (in addition to $p_{f}$ ) to denote the Gelfand transform of $f \in \mathfrak{A}$.

II.3. Remark. Instead of realizing $\widehat{X}$ as the closure of the graph

$$
\left\{\left(x,(f(x))_{f \in \mathfrak{A}}\right): x \in X\right\} \subset X \times \prod_{f \in \mathfrak{A}} \overline{f(X)}
$$

as above, it is also possible to realize $\widehat{X}$ as the closure of the graph $\{(x$, $\left.\left.(f(x))_{f \in E}\right): x \in X\right\} \subset X \times \prod_{f \in E} \overline{f(X)}$ where $E$ is any $\Sigma$-invariant subset of 
the unit ball of $\mathfrak{A}$ which topologically generates $\mathfrak{A}$; i.e., the algebraic span of $E$ is dense in $\mathfrak{A}$.

II.4. Remark. If $E$ has countable cardinality, then $C(Y)$ is separable, so $Y$ is metrizable [2, p. 144, Theorem 6.6]. A metric is given by

$$
d\left(y_{1}, y_{2}\right)=\sum_{n=1}^{\infty} \frac{1}{2^{n}}\left|\widehat{\xi}_{n}\left(y_{1}\right)-\widehat{\xi}_{n}\left(y_{2}\right)\right|
$$

where $E=\left\{\xi_{n}: n=1,2, \ldots\right\}$.

II.5. Remark. The action of $\widehat{\varphi}$ on $Y$ is $\widehat{\varphi}(y)=\left(\varphi(x),\left(t_{f \circ \varphi}\right)_{f \in \mathfrak{A}}\right)$ where $y=$ $\left(x,\left(t_{f}\right)_{f \in \mathfrak{A}}\right.$.

II.6. Definition. Let $f \in B(X), x \in X$, We define the limit set of $f$ at $x$ to be $\left\{z \in \mathbf{C}\right.$ : there exists a net $\left(x_{i}\right) \subset X, x_{i} \rightarrow x, x_{i} \neq x$, such that $\left.f\left(x_{i}\right) \rightarrow z\right\}$, and denote it by $\Lambda(f ; x)$. The same notation will be used for vector-valued functions $\vec{f}: X \rightarrow \mathbf{C}^{n}$.

II.7. Lemma. Let $f \in \mathfrak{A}, x \in X$.

(i) $\hat{f}\left(p^{-1}(x)\right)=\Lambda(f ; x) \cup\{f(x)\}$.

(ii) If $f$ is continuous at $x, \hat{f}\left(p^{-1}(x)\right)=\{f(x)\}$.

Proof. (i) Let $\hat{x} \in p^{-1}(x)$. Then either $\hat{x}=\left(x,(f(x))_{f \in \mathfrak{A}}\right)$, in which case $\hat{f}(\hat{x})=f(x)$, or else $\hat{x}=\lim \left(x_{i},\left(f\left(x_{i}\right)\right)_{f \in \mathfrak{A}}\right)$, where $x_{i} \rightarrow x, x_{i} \neq x$, and $f\left(x_{i}\right) \rightarrow \lambda_{f} \in \Lambda(f ; x)$. (ii) follows immediately from (i).

We pause to motivate the main theorem with the following

II.8. Examples. (i) Let $\chi$ be the characteristic function of an orbit $\left\{\varphi^{k}\left(x_{0}\right): k \in\right.$ Z) ; so $\chi \circ \varphi=\chi$. Take $\mathfrak{A}$ to be the ( $\Sigma$-invariant) $C^{*}$-algebra generated by $C(X)$ and $\chi$. Since $f \rightarrow \hat{f}$ is an isometric isomorphism, $\hat{\chi}^{2}=\widehat{\chi}$ with range $\{0,1\}$; thus $\widehat{X}_{0} \equiv \widehat{\chi}^{-1}(0)$ and $\widehat{X}_{1} \equiv \widehat{\chi}^{-1}(1)$ are nonempty open sets whose union is $\hat{X}$. It follows from $\hat{\chi}=\widehat{\chi \circ \varphi}=\hat{\chi} \circ \widehat{\varphi}$ that $\widehat{X}_{0}$ and $\hat{X}_{1}$ are Zinvariant. In particular, $(\widehat{X}, \widehat{\varphi}, \mathbf{Z})$ is not topologically transitive.

(ii) Suppose $\mathfrak{A}$ contains the function $e_{x_{0}}$. Then $y_{0}=\left(x_{0},\left(f\left(x_{0}\right)\right)_{f \in \mathfrak{A}}\right) \in$ $Y=\widehat{X}$ is an isolated point as is each $\widehat{\varphi}^{k}\left(y_{0}\right), k \in \mathbf{Z}$. It follows that $(\widehat{X}, \widehat{\varphi}, \mathbf{Z})$ is not minimal, for $Y$ being compact implies there is a $y_{1} \in Y \backslash \mathscr{O}_{\hat{\varphi}}\left(y_{0}\right)$. Then $y_{0} \notin \mathscr{O}_{\hat{\varphi}}\left(y_{1}\right)$, so $y_{0} \notin \overline{\mathscr{O}_{\hat{\varphi}}\left(y_{1}\right)}$ since $y_{0}$ is isolated.

II.9. Theorem. Let $(X, \varphi, \Sigma)$ be a dynamical system, and let $\mathfrak{A} \supset C(X)$ be a $\Sigma$ invariant $C^{*}$-algebra which is topologically generated by a subset $E$ of functions, each of which is continuous on a cofinite set. Let $\widehat{X}$ be the spectrum of $\mathfrak{A}$ and $(\widehat{X}, \widehat{\varphi}, \Sigma)$ the resulting extension with $p: \widehat{X} \rightarrow X$ the continuous surjection. Consider the following conditions.

(i) $f(x) \in \Lambda(f ; x)$ for all $f \in \mathfrak{A}$ and for all $x \in X$.

(ii)" $\mathfrak{A}$ contains no characteristic function $e_{x}(x \in X)$. 
(iii) If the orbit $\mathscr{O}_{\varphi}\left(x_{0}\right)$ is dense in $X$, then the orbit $\mathscr{O}_{\hat{\varphi}}\left(\hat{x}_{0}\right)$ is dense in $\hat{X}$ for every $\hat{x}_{0} \in p^{-1}\left(x_{0}\right),\left(x_{0} \in X\right)$.

Conditions (i) and (ii) are equivalent, and each implies (iii). If we also assume that $\varphi^{-1}(x)$ is a finite set for each $x \in X, X$ contains no isolated points, and there is (at least) one point in $X$ with a dense orbit, then (iii) implies (i) and (ii).

Following immediately is the

II.10. Corollary. Suppose $\mathfrak{A}$ satisfies either (i) or (ii). Then,

(a) if $(X, \varphi, \Sigma)$ is topologically transitive, $(\widehat{X}, \widehat{\varphi}, \Sigma)$ is also topologically transitive;

(b) if $(X, \varphi, \Sigma)$ is minimal, $(\widehat{X}, \widehat{\varphi}, \Sigma)$ is also minimal.

Proof of Theorem II.9. Clearly (i) $\Rightarrow$ (ii). Suppose (i) fails to hold, so there is a $g \in \mathfrak{A}$ and $x_{0} \in X$ with $g\left(x_{0}\right) \notin \Lambda\left(g ; x_{0}\right)$. Set

$$
\begin{array}{lll}
g_{1}=g-g\left(x_{0}\right) ; & g_{1}\left(x_{0}\right)=0, & \\
g_{2}=g_{1} \bar{g}_{1} ; & g_{2}\left(x_{0}\right)=0, & \operatorname{range}\left(g_{2}\right) \subset[0, \infty), \\
g_{3}=g_{2} /\left\|g_{2}\right\| ; & g_{3}\left(x_{0}\right)=0, & \operatorname{range}\left(g_{3}\right) \subset[0,1], \\
g_{4}=1-g_{3} ; & g_{4}\left(x_{0}\right)=1, & \operatorname{range}\left(g_{4}\right) \subset[0,1] .
\end{array}
$$

Since $1 \notin \Lambda\left(g_{4} ; x_{0}\right)$, there is an $\varepsilon>0$ and an open neighborhood $U$ of $x_{0}$ such that $g_{4}(x) \leq 1-\varepsilon$ for $x \in U \backslash\left\{x_{0}\right\}$. By Urysohn's lemma there is an $h \in C(X), 0 \leq h \leq 1, h\left(x_{0}\right)=1, \operatorname{supp}(h) \subset U$. Let $g_{5}=h g_{4}$. Then $g_{5} \in \mathfrak{A}$ and $g_{5}\left(x_{0}\right)=1$, while $0 \leq g_{5}(x) \leq 1-\varepsilon$ for $x \in X \backslash\left\{x_{0}\right\}$. Then $e_{x_{0}}=\lim _{n \rightarrow \infty} g_{5}^{n} \in \mathfrak{A}$. Thus (ii) $\Rightarrow$ (i).

To show (i) $\Rightarrow$ (iii) we need the following

II.11. Lemma. Suppose $g\left(x_{0}\right) \in \Lambda\left(g ; x_{0}\right)$ for all $x_{0} \in X$ and for all $g \in$ $C^{*}\left(f_{1}, f_{2}, \ldots, f_{n}\right)$ where $C^{*}\left(f_{1}, f_{2}, \ldots, f_{n}\right)$ is the $C^{*}$-algebra generated by $f_{1}, \ldots, f_{n}$

$\in B(X)$. Then $\vec{f}\left(x_{0}\right) \in \Lambda\left(\vec{f} ; x_{0}\right)$ where $\vec{f}=\left(f_{1}, \ldots, f_{n}\right)$ maps $X$ to $\mathbf{C}^{n}$.

Proof. Suppose $\vec{f}\left(x_{0}\right) \notin \Lambda\left(\vec{f}, x_{0}\right)$. Let $K \subset \mathbf{C}^{n}$ be compact such that $\vec{f}(X) \subset$ $K$. Since $\Lambda\left(\vec{f} ; x_{0}\right)$ is closed, by Urysohn's lemma there is a continuous function $u: K \rightarrow[0,1]$ such that $u\left(\vec{f}\left(x_{0}\right)\right)=0$ and $\left.u\right|_{\Lambda\left(\vec{f}, x_{0}\right)} \equiv 1$.

Consider the coordinate functions $q_{i}: K \rightarrow \mathbf{C}, q_{i}\left(z_{1}, \ldots, z_{n}\right)=z_{i}$. By Stone-Weierstrass, the algebra over $\mathbf{C}$ generated by $\left\{q_{1}, \ldots, q_{n}, \bar{q}_{1}, \ldots, \bar{q}_{n}, 1\right\}$ is dense in the continuous functions on $K$. Hence $h=u\left(f_{1}, \ldots, f_{n}\right) \in$ $C^{*}\left(f_{1}, \ldots, f_{n}\right)$. But then $h\left(x_{0}\right) \notin \Lambda\left(h ; x_{0}\right)$, a contradiction.

(i) $\Rightarrow$ (iii). By Remark II.3 we may consider $\hat{X} \subset X \times \prod_{f \in E} \overline{f(X)}$ where each $f \in E$ is continuous on a cofinite set. Let $U \subset \widehat{X}$ be a nonempty basic 
open set; i.e. given, say $f_{1}, \ldots, f_{n} \in E$, let $U_{i}$ be open in $\overline{f_{i}(X)}$. Then

$$
U=\widehat{X} \cap\left(U^{\prime} \times U^{\prime \prime} \times \prod_{f \in E \backslash\left\{f_{1}, \ldots, f_{n}\right\}} \overline{f(X)}\right)
$$

where $U^{\prime}$ is open in $X$ and $U^{\prime \prime}=U_{1} \times \cdots \times U_{n}$.

Let $\vec{f}=\left(f_{1}, \ldots, f_{n}\right): X \rightarrow \mathbf{C}^{n}$, then $\vec{f}$ is continuous on a cofinite subset $X_{0} \subset X$. Since by Lemma II.11 $\vec{f}$ satisfies the limit set condition, the nonempty set $\vec{f}^{-1}\left(U^{\prime \prime}\right) \cap U^{\prime}$ has infinite cardinality. Thus the set $\vec{f}^{-1}\left(U^{\prime \prime}\right) \cap$ $U^{\prime} \cap X_{0}$ is nonempty. But the latter is $\left(\vec{f} \mid X_{0}\right)^{-1}\left(U^{\prime \prime}\right) \cap U^{\prime} \equiv V$ which is open in $X_{0}$-hence also in $X$-since $\vec{f} \mid X_{0}$ is continuous.

By hypothesis (iii) of the theorem, the orbit of $x_{0}$ is dense in $X$, so there is a $k \in \Sigma$ with $\varphi^{k}\left(x_{0}\right) \in V$. Choose an arbitrary $\hat{x}_{0} \in p^{-1}\left(x_{0}\right)$. Since $p\left(\widehat{\varphi}^{k}\left(\hat{x}_{0}\right)\right) \in V, \widehat{\varphi}^{k}\left(\hat{x}_{0}\right) \in U$, and we conclude that the orbit $\mathscr{O}_{\hat{\varphi}}\left(\hat{x}_{0}\right)$ is dense in $\widehat{X}$.

(iii) $\Rightarrow$ (ii). Suppose $e_{x_{0}} \in \mathfrak{A}$ and $x_{1}$ has a dense orbit in $X$.

Case 1. $x_{0}=x_{1}$. Since $x_{0}$ is not an isolated point, $p^{-1}\left(x_{0}\right)$ contains at least two points $y_{0}$ and $y_{0}^{\prime}$ where $y_{0}=\left(x_{0},\left(f\left(x_{0}\right)\right)_{f \in \mathfrak{A}}\right)$ and

$$
y_{0}^{\prime}=\lim \left(x_{i},\left(f\left(x_{i}\right)\right)_{f \in \mathfrak{A}}\right), \quad x_{i} \rightarrow x_{0}, x_{i} \neq x_{0} .
$$

Since $y_{0}$ is an isolated point, $y_{0} \notin \overline{\mathscr{O}_{\hat{\varphi}}\left(y_{0}^{\prime}\right)}$.

Case 2. $x_{0} \in \mathscr{O}\left(x_{1}\right)$. Then $x_{0}=\varphi^{k}\left(x_{1}\right)$, so $e_{x_{0}} \circ \varphi^{k} \in \mathfrak{A}$ is the characteristic function of the finite set $\varphi^{-k}\left(x_{0}\right)$, which includes the point $x_{1}$. By Urysohn's lemma, there is a function $h \in C(X)$ such that $h\left(x_{1}\right)=1$ and $\left.h\right|_{\varphi^{-k}\left(x_{0}\right) \backslash x_{1}} \equiv 0$. Then $h \cdot\left(e_{x_{0}} \circ \varphi^{k}\right)=e_{x_{1}} \in \mathfrak{A}$. This is Case 1 .

Case 3. $x_{0} \notin \mathscr{O}_{\varphi}\left(x_{1}\right)$. Since $y_{0}$ is isolated, $y_{0} \notin \overline{\mathscr{O}_{\hat{\varphi}}\left(\hat{x}_{1}\right)}$ for any $\hat{x}_{1} \in$ $p^{-1}\left(x_{1}\right)$.

II.12. Corollary. Let $(X, \varphi, \Sigma)$ be a dynamical system such that $X$ has no isolated points and $\varphi$ is an open map. Let $\left\{x_{i}\right\}_{i \in I} \subset X$ be a collection of points and $\left\{\xi_{i}\right\}_{i \in I} \subset B(X)$ a collection of functions satisfying

(a) $\xi_{i}$ is continuous on $X \backslash\left\{x_{i}\right\}, i \in I$;

(b) the orbits $\mathscr{\mathscr { O }}_{\varphi}\left(x_{i}\right), \mathscr{O}_{\varphi}\left(x_{j}\right)$ are disjoint for $i \neq j$;

(c) $\varphi^{-k}\left(x_{i}\right)$ is a finite set for $k \in \mathbf{N}, i \in I$.

Let $\mathfrak{A}$ be the $C^{*}$-subalgebra of $B(X)$ generated by $C(X)$ and the functions $\xi_{i} \circ \varphi^{k}, i \in I, k \in \Sigma$. Then Theorem II.9 applies to $\mathfrak{A}$, and condition (i) may be rephrased as:

$$
\xi_{i}\left(x_{i}\right) \in \Lambda\left(\xi_{i} ; x_{i}\right) \text { for all } i \in I \text {. }
$$


Proof. If $\varphi$ is a homeomorphism, $\xi_{i} \circ \varphi^{k}$ has at most one point of discontinuity, $\varphi^{-k}\left(x_{i}\right)$, and clearly $\xi_{i}$ satisfies the limit set condition at $x_{i}$ iff $\xi_{i} \circ \varphi^{k}$ satisfies the condition at $\varphi^{-k}\left(x_{i}\right)$. Suppose $\Sigma=\mathbf{N}$ and $\xi_{i}\left(x_{i}\right) \in \Lambda\left(\xi_{i} ; x_{i}\right)$. Let $\left\{t_{n}\right\}$ be a net converging to $x_{i}$ such that $\xi_{i}\left(x_{i}\right)=\lim _{n} \xi_{i}\left(t_{n}\right)$. For a fixed $k \in \mathbf{N}$ let $s \in \varphi^{-k}\left(x_{i}\right)$. Given an open neighborhood, $U_{j}$, of $s, \varphi^{k}\left(U_{j}\right)$ is an open neighborhood of $x_{i}$, so there is an $s_{j} \in U_{j}$ such that $\varphi^{k}\left(s_{j}\right)=t_{n_{j}}$. Then the net $s_{j} \rightarrow s$ and $\lim _{j} \xi_{i} \circ \varphi^{k}\left(s_{j}\right)=\lim _{j} \xi_{i}\left(t_{n_{j}}\right)=\lim _{n} \xi_{i}\left(t_{n}\right)=\xi_{i}\left(x_{i}\right)=\xi_{i} \circ \varphi^{k}(s)$. It follows from this argument and (b) that $f\left(x_{i}\right) \in \Lambda\left(f ; x_{i}\right) \forall f \in \mathfrak{A}, \forall i \in I$. Since $X$ has no isolated points, $f(x) \in \Lambda(f ; x) \forall f \in \mathfrak{A}$ and $\forall x \in X$.

Finally, it follows from condition (c) that the functions $\xi_{i} \circ \varphi^{k}$, which together with $C(X)$ topologically generate $\mathfrak{A}$, are each continuous on a cofinite set.

II.13. Remark. It would be interesting to have a theorem analogous to Theorem II.9 which applied to arbitrary $\Sigma$-invariant $C^{*}$-function algebras between $C(X)$ and $B(X)$. Indeed, the equivalence of conditions (i) and (ii) does hold in general. However, without some restriction (i) does not imply (iii). For let $(X, \varphi, \mathbf{Z})$ be a minimal dynamical system and take $(\widehat{X}, \widehat{\varphi}, \mathbf{Z})$ to be the extension described in Example II.8(i). This system is not even topologically transitive even though the limit set condition is satisfied for every $f \in \mathfrak{A}$.

Finally, we see that an extension can be topologically transitive even if condition (i) of Theorem II.9 is not satisfied.

II.14. Proposition. Let $(X, \varphi, \Sigma)$ be a dynamical system and let $\left\{x_{i}\right\},\left\{\xi_{i}\right\}$ be as in Corollary II.12. Fix an index $i_{0}=0 \in I$, and assume that $\xi_{0}\left(x_{0}\right) \notin$ $\Lambda\left(\xi_{0} ; x_{0}\right)$; otherwise assume $\xi_{i}\left(x_{i}\right) \in \Lambda\left(\xi_{i} ; x_{i}\right)$. Suppose that $\mathscr{O}_{\varphi}\left(x_{0}\right)$ is dense in $X$, and let $y_{0}=\left(x_{0},\left(f\left(x_{0}\right)\right)_{f \in \mathfrak{A}}\right)$. Then,

(i) if $\Sigma=\mathbf{N}, y_{0}$ is the unique point of $\widehat{X}$ with a dense orbit;

(ii) if $\Sigma=\mathbf{Z}$, then $y$ has a dense orbit iff $y \in \mathscr{O}_{\hat{\varphi}}\left(y_{0}\right)$.

Sketch of Proof. Since the points $\mathscr{O}_{\hat{\varphi}}\left(y_{0}\right)$ are open, no point lying outside this orbit can itself have a dense orbit. The fact that $\mathscr{O}_{\hat{\varphi}}\left(y_{0}\right)$ is dense is essentially the same as in Theorem II.9.

\section{REFERENCES}

1. S. K. Berberian, The character space of the algebra of regulated functions, Pacific J. Math. 741 (1978), 15-35.

2. J. B. Conway, A course in functional analysis, Springer-Verlag, New York, 1985.

3. J. Dixmier, $C^{*}$-algebras, North-Holland, Amsterdam, 1977.

4. R. Ellis, Lectures on topological dynamics, Benjamin, New York, 1969.

5. $\mathrm{H}$. Furstenberg, Recurrence in ergodic theory and combinatorial number theory, Princeton Univ. Press, Princeton, N. J., 1981.

6. P. Walters, An Introduction to ergodic theory, Springer-Verlag, New York, 1982.

Department of Mathematics, Hope College, Holland, Michigan 49423

Department of Mathematics, Iowa State University, Ames, Iowa 50011 